\title{
Relationship between Daytime Sleepiness and Intrinsically Photosensitive Retinal Ganglion Cells in Glaucomatous Disease
}

\author{
Carolina P. B. Gracitelli, ${ }^{1}$ Gloria Liliana Duque-Chica, ${ }^{2}$ Ana Laura de Araújo Moura, ${ }^{1,2}$ \\ Marina Roizenblatt, ${ }^{1}$ Balazs V. Nagy, ${ }^{2}$ Geraldine Ragot de Melo, ${ }^{1}$ Paula Delegrego Borba, ${ }^{1}$ \\ Sérgio H. Teixeira, ${ }^{1}$ Sergio Tufik, ${ }^{3}$ Dora Fix Ventura, ${ }^{2}$ and Augusto Paranhos Jr. ${ }^{1}$ \\ ${ }^{1}$ Department of Ophthalmology, Federal University of São Paulo, Rua Botucatu, 821 Vila Clementino, 04023-062 São Paulo, SP, Brazil \\ ${ }^{2}$ Experimental Psychology Department, Institute of Psychology, University of São Paulo, São Paulo, SP, Brazil \\ ${ }^{3}$ Sleep Medicine Division, Psychobiology Department, Federal University of São Paulo, São Paulo, SP, Brazil
}

Correspondence should be addressed to Carolina P. B. Gracitelli; carolepm@gmail.com

Received 5 November 2015; Revised 2 January 2016; Accepted 12 January 2016

Academic Editor: Antonio Benito

Copyright (C) 2016 Carolina P. B. Gracitelli et al. This is an open access article distributed under the Creative Commons Attribution License, which permits unrestricted use, distribution, and reproduction in any medium, provided the original work is properly cited.

\begin{abstract}
Patients with glaucoma showed to have higher daytime sleepiness measured by Epworth sleepiness scale. In addition, this symptom was associated with pupillary reflex and polysomnography parameters. These ipRGC functions might be impaired in patients with glaucoma, leading to worse quality of life.
\end{abstract}

\section{Introduction}

The hypothalamic suprachiasmatic nucleus, the primary circadian oscillator in humans, is the site for the master circadian clock $[1,2]$. Light phase shifts the endogenous oscillator in the hypothalamic suprachiasmatic nucleus, synchronizing it with the day-night cycle $[1,2]$. Some years ago, a study of human eyes culminated in the breakthrough discovery of intrinsically photosensitive retinal ganglion cells (ipRGCs), which constitute a subtype of retinal ganglion cells (RGCs) that express melanopsin, a photopigment that is most sensitive to short-wavelength blue light $(480 \mathrm{~nm})$ $[3,4]$. In addition to being intrinsically light-sensitive $[3,5-$ 7], ipRGCs are associated with circadian, neuroendocrine, and neurobehavioral functions as well as having influence on some image-forming functions [6-9].

Glaucoma, an optic neuropathy characterized by the progressive loss of RGCs, is associated with morphological changes in the optic nerve and the retinal nerve fiber layer (RNFL) [10]. Several investigators have explored whether light transmission to the hypothalamic suprachiasmatic nucleus or the postillumination pupillary light response (PLR) is compromised as a result of ipRGC damage in glaucoma [11-14]. The activity of ipRGCs has been evaluated in patients with glaucoma using either the light-induced reduction of nocturnal pineal melatonin secretion or the PLR test as a functional indicator of melanopsin-based phototransduction [12-14]. Most of these previous studies have shown significant reductions in ipRGC function in affected eyes compared with the eyes of healthy subjects [11-14]. In addition, previous studies using the PLR test have demonstrated significant correlations between decreased ipRGC function and functional [11, 13] and structural [15] damage in patients with glaucoma.

Few studies have tried to find an association between abnormal ipRGC function and the severity of sleep disorders in glaucoma patients $[16,17]$. Wang et al. used the self-rated Pittsburgh Sleep Quality Index (PSQI) to evaluate whether glaucoma affects sleep quality [16]. These authors showed that the prevalence of sleep disorders was higher among patients with glaucoma than among healthy participants [16]. Although these studies indicate that patients with glaucoma exhibit significantly altered sleep patterns that are also correlated with the PLR test, the relationships between objective polysomnographic measures and self-reported diurnal symptoms (e.g., excessive daytime sleepiness) have yet to be 
established. Furthermore, daytime sleepiness has not been associated with ipRGC functions such as PLR.

The degree of daytime sleepiness can be assessed using the Epworth sleepiness scale (ESS), which can be helpful in diagnosing sleep disorders [18]. The ESS score has never been determined in a glaucomatous population, nor has it been associated with the objective polysomnography measures that are considered as the gold-standard test for assessing sleep disorders. The study uses the PLR (as a parameter for ipRGC dysfunction) and correlated these measurements with ESS and polysomnography to evaluated correlations between sleep disorder and ipRGC function.

\section{Methods}

The protocol for this cross-sectional study followed the tenets of the Declaration of Helsinki and was approved by the Institutional Review Board of the Federal University of São Paulo (CEP 262.470). Written informed consent was obtained from all subjects.

2.1. Study Sample. A total of 40 participants were recruited from the Federal University of São Paulo. Thirty of these participants had primary open-angle glaucoma, and 10 others were included for the control group. The inclusion and exclusion criteria, as well as the ophthalmological assessments, have been previously used and are described in detail as follows [15,17]. All subjects underwent a complete ophthalmological examination, including a medical history review, best-corrected visual acuity measurement, slit-lamp biomicroscopy, gonioscopy, intraocular pressure (IOP) measurement, dilated fundoscopic examination using a 78-diopter (D) lens, refraction, and standard automated perimetry (SAP) using the Swedish interactive threshold algorithm (SITA Standard 24-2; Carl Zeiss Meditec, Inc., Dublin, CA, USA). Subjects were excluded if they were younger than 40 years old or older than 80 years old; had a best-corrected visual acuity worse than $0.2 \log$ MAR (logarithm of the minimum angle of resolution); had a previous history of ophthalmic surgery; had lens opacity greater than 0.5 (cortical opacity, nuclear opalescence, posterior subcapsular opacity, or changes in nuclear color) according to the Lens Opacity Classification System III (LOCS III) [19]; had any corneal, retinal, or orbital diseases; presented with an absolute spherical refractive error $>5 \mathrm{D}$ or an absolute cylindrical error $>3 \mathrm{D}$; or were using alpha-adrenergic agonist eye drop medication or any systemic medication that might affect PLR. Participants with a history of taking psychoactive agents were excluded from the study. In addition, none of our patients reported any travel or shift work during the examination period. Only patients with an open angle at gonioscopy were included in our study.

The participants were considered as having glaucoma if they had at least 3 repeatable, consecutive, abnormal visual field test results, which were defined as a pattern standard deviation outside the $95 \%$ confidence interval for the normal range or a glaucoma hemifield test result outside the normal limits and a corresponding alteration in the appearance of the optic disc. Patients were also considered as having glaucoma if they showed signs of glaucomatous optic neuropathy at clinical examination by a glaucoma expert, confirmed by stereophotography. Glaucomatous damage to the optic disc nerve was defined as the presence of RNFL defects or localized or diffuse neuroretinal rim loss.

The control participants showed normal results at ophthalmological examination, an IOP of $<21 \mathrm{mmHg}$, normal visual field test results, and absence of glaucomatous optic neuropathy based on fundoscopic and stereoscopic optic disc photograph evaluations.

The entire cohort (i.e., the 30 patients with primary open-angle glaucoma and the 10 controls) belongs to the same sample of a study that was published elsewhere [15, 17]. No additional participant was recruited to augment that previously published sample $[15,17]$.

2.2. Sleep Disorder Questionnaire. Sleep disorders were evaluated using the ESS, which has been widely used as a subjective measurement of daytime sleepiness [18]. This test measures the probability of falling asleep across eight daily situations; each question is scored as 0 (e.g., would never doze), 1 (slight change of dozing), 2 (moderate chance of dozing), or 3 (high chance of dozing), for a total possible score of 24 . The clinically normal score of this scale ranges from 2 to 10. A score higher than 10 indicates a borderline or increased level of chronic sleepiness, and a score higher than 15 is defined as excessive daytime sleepiness [18].

2.3. PLR Test. Measurement of the PLR was based on a method developed by Park et al. [20]; our previous studies $[15,17]$ and other research groups have used this technique [21]. To preferentially stimulate ipRGC function, we used $470 \mathrm{~nm}$ (blue) flashes with luminance of $250 \mathrm{~cd} / \mathrm{m}^{2}(14.1 \mathrm{log}$ photons $/ \mathrm{cm}^{2} / \mathrm{s}$ melanopic excitation) [2] of $1 \mathrm{~s}$ in duration. Alternatively, to preferentially stimulate different retinal photoreceptors (e.g., cones and rods) without directly stimulating the ipRGCs, we used $640 \mathrm{~nm}$ (red) flashes of $1 \mathrm{~s}$ in duration with luminance of $250 \mathrm{~cd} / \mathrm{m}^{2}\left(10.7 \mathrm{log}\right.$ photons $/ \mathrm{cm}^{2} / \mathrm{s}$ melanopic excitation) [2]. Table 1 summarizes full equations for calculating illuminance values for ipRGC, cones, and rods functions [2]. For each stimulus, a red flash was presented first, followed by a blue flash $60 \mathrm{~s}$ after the offset of the red flash. The interval between stimuli was used to allow the pupil size to return to baseline before the presentation of the subsequent stimulus. Both eyes were tested monocularly in a random order. One pupillometric measurement per participant was performed. The patients were dark-adapted for $10 \mathrm{~min}$; then, alternating $1 \mathrm{~s}$ red and blue flashes were presented at luminance of $250 \mathrm{~cd} / \mathrm{m}^{2}$ (10.7 and $14.1 \mathrm{log}$ photons $/ \mathrm{cm}^{2} / \mathrm{s}$ melanopic excitation, resp.) [2].

Stimuli were generated by corresponding light-emitting diodes (LEDs) in a Ganzfeld system (RETIport; Roland Consult, Brandenburg, Germany), and responses were recorded using an eye-tracking camera system with an infrared LED (IR333-A; Arrington Research, Scottsdale, AZ, USA). The Arrington Research infrared camera recorded the procedure at 60 frames per second. The peak wavelength of the infrared 
TABLE 1: Photometric measures for different photoreceptors' inputs to circadian and neurophysiological light responses in humans [2].

\begin{tabular}{|c|c|c|c|}
\hline Photoreceptor & Photopigment & Spectral sensitivity function & Unit of measure $^{\mathrm{a}}$ \\
\hline Short-wavelength (S) cones & $\begin{array}{l}\text { S-cone photopsin } \\
\text { (cyanolabe) }\end{array}$ & Cyanolabe response function $N_{\mathrm{sc}}(\lambda)$ & $\begin{array}{l}\text { Cyanopic illuminance } \\
\text { (cyanopic-lux) }\end{array}$ \\
\hline Medium-wavelength (M) cones & $\begin{array}{l}\text { M-cone photopsin } \\
\text { (chlorolabe) }\end{array}$ & Chlorolabe response function $N_{\mathrm{mc}}(\lambda)$ & $\begin{array}{l}\text { Chlorolabe illuminance } \\
\text { (chloropic-lux) }\end{array}$ \\
\hline Long-wavelength $(\mathrm{L})$ cones & $\begin{array}{l}\text { L-cone photopsin } \\
\text { (erythrolabe) }\end{array}$ & Erythrolabe response function $N_{\mathrm{lc}}(\lambda)$ & $\begin{array}{l}\text { Erythrolabe illuminance } \\
\quad \text { (erythropic-lux) }\end{array}$ \\
\hline $\begin{array}{l}\text { ipRGCs (intrinsic } \\
\text { photosensitivity) }\end{array}$ & Melanopsin & Melanopsin response function $N_{z}(\lambda)$ & $\begin{array}{l}\text { Melanopsin illuminance } \\
\text { (melanopic-lux) }\end{array}$ \\
\hline Rods & Rod opsin & Rod opsin response function $N_{r}(\lambda)$ & $\begin{array}{l}\text { Rhodopic illuminance } \\
\text { (rhodopic-lux) }\end{array}$ \\
\hline
\end{tabular}

$\mathrm{a}=$ each unit of measure (Ea, where a specifies the retinal photopigment) is derived by convoluting the spectral power distribution of incident light (Eel) with the relevant spectral sensitivity function, which in turn is defined by the photopigment spectral sensitivity adjusted for prereceptoral filtering in a standard observer $(\mathrm{Na}(\mathrm{l})$; see the online reference (http://www.cie.co.at/index.php?i_ca_id=983) [28] for full functions and a detailed description of their derivation) according to the equation $\mathrm{Ea}=72983.25 \mathrm{R} \mathrm{Eel}(\mathrm{l}) \mathrm{Na}(\mathrm{l}) \mathrm{dl}$. Species-specific variants of the spectral sensitivity functions may be required for nonhuman applications to account for differences in prereceptoral filtering and photopigment spectral sensitivity [2].

LEDs was $940 \mathrm{~nm}[20,22,23]$. Pupil size was directly measured; the largest diameter was considered as the primary measure. The peak PLR amplitude was calculated as the maximal pupillary constriction and expressed relative to the baseline value (peak PLR amplitude $=$ maximal constriction diameter/baseline diameter) [20]. In addition, the sustained PLR was expressed as the pupil diameter $6 \mathrm{~s}$ after the flash offset relative to baseline [20]. The absolute values of the peak and sustained responses to blue and red flashes at $250 \mathrm{~cd} / \mathrm{m}^{2}$ for a similar cohort were reported in our previous studies $[15,17]$.

2.4. Polysomnography. To obtain sleep parameter data, polysomnographic recordings were performed over a full night in a temperature-controlled and sound-attenuated room (EMBLA_S7000, Embla Systems Inc., Broomfield, CO, USA). The details of this methodology have been described and used previously elsewhere [17].

The following parameters were included in the polysomnographic assessment [24]: total sleep time (TST; the amount of actual sleep time over a sleep period); sleep efficiency (TST per sleep period); sleep latency (i.e., the amount of time before starting the effective sleep period); REM sleep latency (i.e., the amount of time before starting REM sleep); and the S1 to S3 and REM sleep stages (as a percentage of TST) [25]. Arousal was defined as a $\geq 3 \mathrm{~s}$ increase in the electroencephalographic frequency preceded by $\geq 10 \mathrm{~s}$ of stable sleep [25]. The following data were collected for the arousal assessment: total arousal duration after falling asleep (in minutes), total number of arousals ( $n$ ), and arousal index (number of events per hour) [25]. The apnea-hypopnea index (AHI, event count per hour of TST) was calculated as the total number of episodes of apnea and hypopnea per hour of electroencephalographically confirmed sleep. Apnea was defined as the cessation of airflow for at least $10 \mathrm{~s}$ combined with the effort to breathe. Hypopnea was classified as a minimum $30 \%$ reduction in thoracoabdominal movement or airflow compared with baseline lasting at least $10 \mathrm{~s}$ together with $\geq 4 \%$ oxygen desaturation [26]. Our previous study reported different polysomnographic parameters from a similar cohort $[15,17]$.

A board-certified physician who was unaware of the study design (and was therefore blind to patients' ophthalmic examination results) analyzed the polysomnographic tests. The polysomnographic tests were performed within 6 months of the administration of the ESS questionnaire, the ophthalmological exams, and the PLR tests. The ophthalmological exams and the PLR tests were performed within a 1-month interval.

2.5. Statistical Analyses. Both eyes of each participant were included in this analysis. To correct for the bias introduced by the expected correlation between the two eyes of each participant, a generalized estimating equation (GEE) was used to adjust for intereye correlations [27]. After adjusting for within-patient intereye correlations, the GEE was used to examine the relationship between the ESS score and the PLR across the entire cohort. In addition, a linear regression was used to determine the association between the ESS score and the polysomnographic parameters for both groups [27].

To obtain the correlation coefficients regarding the association between the ESS score and the PLR as well as the polysomnographic parameters, a linear regression was performed. Whenever both eyes were eligible, the right eye was arbitrarily selected for this specific analysis. Participant age was examined as a covariate.

Statistical analyses were performed using commercially available Stata software (version 13, StataCorp LP, College Station, TX, USA). The $\alpha$ level (type I error) was set at 0.05 .

\section{Results}

This study examined both eyes of 40 subjects: 30 with glaucoma and 10 healthy (control group). The mean ( \pm standard deviation) ages of the control and glaucoma groups were $56.10 \pm 8.08$ (range $=45$ to 74$)$ and $62.00 \pm 9.39($ range $=42$ to 75$)$ years, respectively $(p=0.084)$. The demographic and clinical characteristics of the participants are summarized in 
TABle 2: Demographic and clinical characteristics (mean \pm standard deviation [median, interquartile range]) of control and glaucoma participants.

\begin{tabular}{|c|c|c|c|}
\hline & $\begin{array}{c}\text { Control group }(N=20 \text { eyes of } 10 \\
\text { subjects })\end{array}$ & $\begin{array}{c}\text { Glaucoma group }(N=60 \text { eyes of } 30 \\
\text { subjects })\end{array}$ & $p$ value \\
\hline Age, years & $\begin{array}{c}56.10 \pm 8.08 \\
(55.50,54.00 \text { to } 58.00)\end{array}$ & $\begin{array}{c}62.00 \pm 9.39 \\
(64.50,53.00 \text { to } 70.00)\end{array}$ & $0.084^{\mathrm{a}}$ \\
\hline Female, $\%$ & $8(80 \%)$ & $20(67 \%)$ & $0.693^{\mathrm{c}}$ \\
\hline Ancestry, \% & & & $0.401^{\mathrm{c}}$ \\
\hline Caucasian & $9(90 \%)$ & $21(70 \%)$ & \\
\hline African-American & $1(10 \%)$ & $9(30 \%)$ & \\
\hline MD worse eye, $\mathrm{dB}$ & $\begin{array}{c}-1.28 \pm 2.03 \\
(-1.23,-3.10 \text { to } 0.44)\end{array}$ & $\begin{array}{c}-11.82 \pm 10.68 \\
(-7.15,-20.22 \text { to }-3.07)\end{array}$ & $<0.001^{b}$ \\
\hline MD better eye, $\mathrm{dB}$ & $-0.04 \pm 1.60(0.27,-1.18$ to 1.52$)$ & $-7.00 \pm 7.97(-2.90,-11.78$ to -1.34$)$ & $<0.001^{\mathrm{b}}$ \\
\hline Average IOP, $\mathrm{mmHg}$ & $13.42 \pm 1.87(13.00,12.00$ to 14.00$)$ & $16.90 \pm 2.82(16.00,15.00$ to 18.00$)$ & $<0.001^{\mathrm{b}}$ \\
\hline Cup/disc ratio & $0.34 \pm 0.10(0.30,0.30$ to 0.40$)$ & $0.80 \pm 0.15(0.80,0.70$ to 1.00$)$ & $<0.001^{\mathrm{b}}$ \\
\hline $\mathrm{CCT}, \mu \mathrm{m}$ & $\begin{array}{c}539.95 \pm 30.24 \\
(534.00,519.00 \text { to } 562.00)\end{array}$ & $\begin{array}{c}521.76 \pm 34.52(520.50,498.00 \text { to } \\
547.00)\end{array}$ & $0.069^{\mathrm{a}}$ \\
\hline $\begin{array}{l}\text { Visual acuity, } \\
\text { logMAR }\end{array}$ & $0.04 \pm 0.07(0.00,0.00$ to 0.10$)$ & $0.11 \pm 0.09(0.10,0.00$ to 0.20$)$ & $<0.001^{\mathrm{a}}$ \\
\hline
\end{tabular}

Table 2. Relevant variation was present with regard to the average $\mathrm{MD}$ in the glaucomatous eyes, ranging from -32.28 to $0.53 \mathrm{~dB}$.

3.1. Overall ESS Results. The mean ESS score of the glaucoma patients was significantly higher than that of the control group $(13.10 \pm 5.14$ and $9.10 \pm 3.73$, resp., $p=0.029)$. With regard to sample size, nineteen glaucoma patients $(63.33 \%)$ and three control patients $(30.00 \%)$ reported an ESS score $>10$, which is considered as borderline or indicative of an increased level of chronic sleepiness; however, this difference was not significant ( $p=0.234$; Fisher's exact test). The ESS score was greater than 15 for 10 of the participants in the glaucoma group (33.33\%), suggesting a high level of daytime sleepiness; however, none of the participants in the control group had an ESS score greater than 15. Figure 1 illustrates the distribution of the ESS scores among the controls and glaucoma patients.

A significant association was found between the ESS score and glaucoma severity based on SAP MD $(p<0.001)$; specifically, higher ESS scores were related to worse damage (Figure 2(a)).

3.2. Association between Polysomnography and ESS Results. Table 3 shows the associations between the ESS scores and the polysomnographic parameters for the entire cohort. A significant inverse correlation was observed between the ESS score and sleep efficiency $(p=0.002)$, indicating that higher ESS scores were associated with lower sleep efficiencies. A significant association was also found between the ESS score and arousal duration after falling asleep $(p<$ 0.001); specifically, higher ESS scores were related to longer

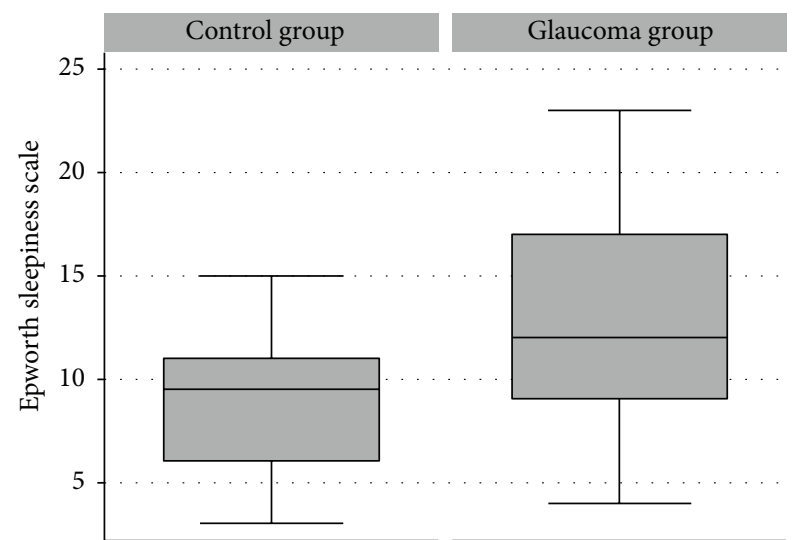

FIGURE 1: Boxplot depicting the distribution of the ESS scores in the control and glaucoma groups. Box: median and interquartile range (IQR). The whiskers show the maximal and minimal 1.5 IQR.

arousal durations after falling asleep. The ESS score was also associated with the number of arousals and the arousal index ( $p=0.039$ and $p=0.013$, resp.). Figure $2(\mathrm{~b})$ illustrates the association between the ESS score and the number of arousals at polysomnography for the entire cohort. Age was included in the multivariate model but did not exert a significant effect ( $p>0.05$ for all polysomnographic parameters).

3.3. Association between PLR Test and ESS Results. A significant inverse correlation was observed between the ESS score and the peak response to the blue flash with a luminance of $250 \mathrm{~cd} / \mathrm{m}^{2}\left(p=0.017, R^{2}=0.080\right)$. Furthermore, a significant correlation was found between the ESS score and 


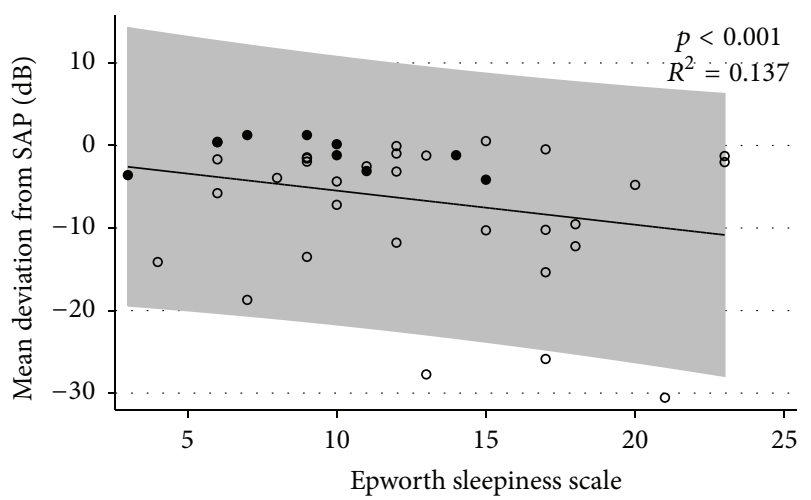

- Control

- Glaucoma

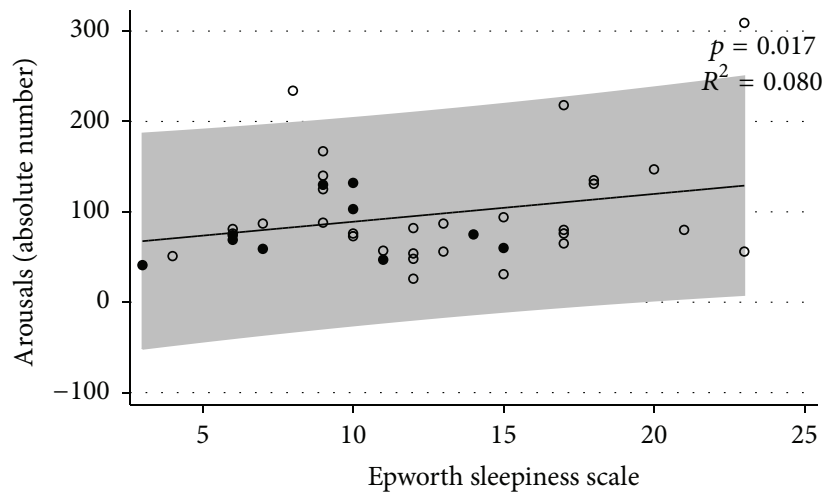

- Control

- Glaucoma

(a)

(b)

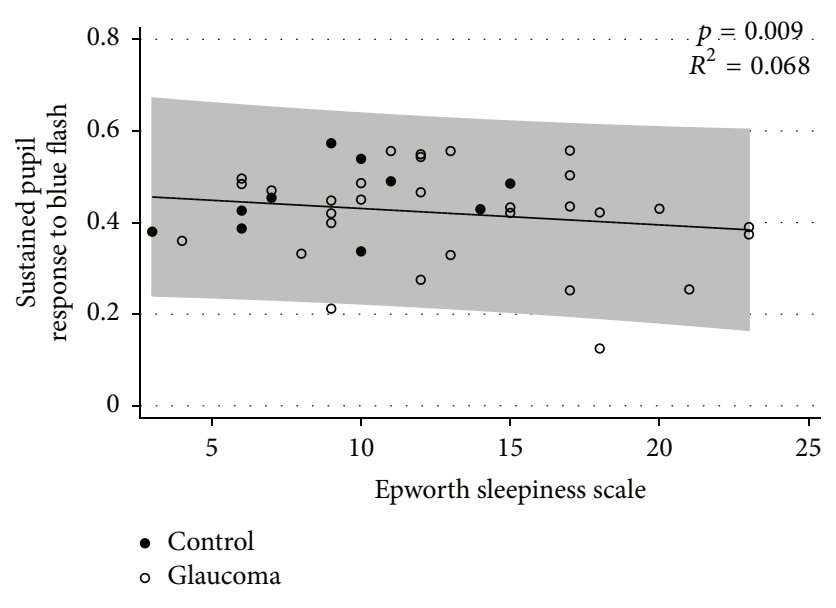

(c)

FIGURE 2: Scatterplot depicting the negative association (a) between the SAP MD and the ESS scores, the positive association between the arousal parameters at polysomnography and the ESS scores (b), and the negative relationship between the sustained pupillary response to the blue flash at $250 \mathrm{~cd} / \mathrm{m}^{2}$ and the ESS score (c) in the control and glaucoma groups. The shaded area represents the prediction interval of the regression.

TABLE 3: Associations $\left(R^{2}\right)$ between the polysomnographic parameters and the ESS scores*.

\begin{tabular}{lcc}
\hline Parameter & $p$ value & $R^{2}$ \\
\hline Arousal duration after falling asleep $(\mathrm{min})$ & $<\mathbf{0 . 0 0 1}$ & 0.252 \\
Sleep efficiency $(\%)$ & $\mathbf{0 . 0 0 2}$ & 0.214 \\
Arousals $(n)$ & $\mathbf{0 . 0 3 9}$ & 0.163 \\
Arousal index $(n / \mathrm{h})$ & $\mathbf{0 . 0 1 3}$ & 0.133 \\
TST (min) & 0.050 & 0.159 \\
Sleep latency (min) & 0.592 & 0.120 \\
REM sleep latency (min) & 0.667 & 0.119 \\
Sleep stage $(\%)$ & & \\
$\quad$ S1 & 0.848 & 0.118 \\
S2 & 0.057 & 0.157 \\
S3 & 0.150 & 0.140 \\
REM & 0.782 & 0.118 \\
\hline
\end{tabular}

* Linear regression model including the polysomnographic parameters and the ESS scores.

Significant values are in boldface. the sustained response to the blue flash with luminance of $250 \mathrm{~cd} / \mathrm{m}^{2}\left(p=0.009, R^{2}=0.068\right)$. A higher ESS score was associated with lower peak and sustained responses to the blue flash at $250 \mathrm{~cd} / \mathrm{m}^{2}$. Figure 2 (c) shows the association between the ESS score and the sustained response to the blue flash with luminance of $250 \mathrm{~cd} / \mathrm{m}^{2}$ for the entire cohort.

An inverse association was also found between the ESS score and the sustained response to the red flash at $250 \mathrm{~cd} / \mathrm{m}^{2}$ $\left(10.7 \log\right.$ photons $\left./ \mathrm{cm}^{2} / \mathrm{s}^{2}\right)\left(p=0.010 ; R^{2}=0.080\right)$; specifically, a higher ESS score was associated with a lower sustained response to the red flash with luminance of $250 \mathrm{~cd} / \mathrm{m}^{2}(10.7$ $\log$ photons $/ \mathrm{cm}^{2} / \mathrm{s}$ ). However, no correlation was observed between the ESS score and the peak response to the red flash at $250 \mathrm{~cd} / \mathrm{m}^{2}\left(10.7 \log\right.$ photons $\left./ \mathrm{cm}^{2} / \mathrm{s} ; p=0.199 ; R^{2}=0.021\right)$.

In addition, age was examined in the multivariate model; however, it was found not to exert a significant effect on the associations between the ESS score and either the peak or sustained response to the blue flash with luminance of $250 \mathrm{~cd} / \mathrm{m}^{2}$ for either the control or glaucoma group 
( $p=0.403$ and $p=0.290$, resp.). In addition, age did not significantly affect the associations between the ESS score and either the peak or sustained response to the red flash with luminance of $250 \mathrm{~cd} / \mathrm{m}^{2}$ for either the control or glaucoma group ( $p=0.223$ and $p=0.375$, resp.).

\section{Discussion}

This study used the ESS to evaluate sleep disorders, specifically daytime sleepiness, in glaucoma patients. In addition, we addressed the relationship between the ESS score and both polysomnographic measures and PLR in glaucoma patients. Increased daytime sleepiness, as measured by the ESS, was associated with arousal duration after falling asleep, sleep efficiency, number of arousals, and the arousal index at polysomnography. In addition, increased ESS scores were associated with reduced peak and sustained PLRs to a blue flash with luminance of $250 \mathrm{~cd} / \mathrm{m}^{2}$. To the best of our knowledge, this study is the first to identify the associations between daytime sleepiness and polysomnography and PLR parameters. Sleep disorders (evaluated using ESS and polysomnography) and PLR might be related to certain types of ipRGCs [29].

The results of this study are in agreement with those of previous studies showing that glaucoma is associated with a decrease in sleep quality $[16,30,31]$. Wang et al. used the PSQI to investigate whether glaucoma affects sleep quality and to evaluate the differences between disparate types of glaucoma (i.e., primary open-angle glaucoma and primary closed-angle glaucoma) [16]. These authors found that the prevalence of sleep disorders was higher among patients with glaucoma and that the patients with primary closed-angle glaucoma had a greater incidence of sleep disorders than those with primary open-angle glaucoma [16]. Using the ESS, we demonstrated that patients with glaucoma showed increased daytime sleepiness. The ESS is a self-report questionnaire that is considered as the gold standard for assessing daytime sleepiness [18]; however, to date, the ESS score has never been used to investigate glaucoma patients. We hypothesized that ocular damage in patients with glaucoma affects all types of RGCs (including ipRGCs) and that this damage is linked to the dysregulation of the circadian system, thereby leading to decreased sleep quality and resulting in excessive daytime sleepiness.

The finding in our study supported these hypotheses, showing that patients with glaucoma have higher daytime sleepiness associated with worse parameters in polysomnography. The excessive daytime sleepiness observed among glaucoma patients was likely because of the increased number or total duration of arousals after falling asleep, decreased sleep efficiency, and briefer TST. Daytime sleepiness (as measured using the ESS) was also associated with different polysomnographic parameters such as sleep efficiency, arousal duration after falling asleep, arousals, and the arousal index. A previous study performed by our group demonstrated that patients with glaucoma have significantly lower average TSTs and sleep efficiencies as well as longer arousal durations after falling asleep [17].
Daytime sleepiness was also associated with PLR, a wellknown indicator of the afferent input from the retina and the optic nerve. Most optic neuropathies show an association between PLR and disease severity [32-36]. Briefly, the PLR shows a transient phase of pupillary constriction, which is attributed to rod and cone input to the ipRGCs, followed by sustained pupil constriction, which is primarily driven by the melanopsin response in the ipRGCs [20,37]. Previous studies have shown an inverse correlation between sustained pupillary constriction and glaucoma severity [11, 13, 15]. For example, Gracitelli et al. showed that the sustained response to the blue flash at $250 \mathrm{~cd} / \mathrm{m}^{2}$ (which preferentially stimulates ipRGCs $[3,4]$ ) was reduced in patients with advanced glaucoma (i.e., patients with SAP MD worse than $-12 \mathrm{~dB}$ ) [15]. In addition, a thinner RNFL was associated with a reduced sustained pupillary response [15]. The present study adds new information by showing that, in addition to the decreased sustained pupillary response in glaucoma patients, the degree of daytime sleepiness is associated with a reduced sustained response to a blue flash at $250 \mathrm{~cd} / \mathrm{m}^{2}$. One possible explanation for this finding is that ipRGCs are among the cells in the total RGC population that are damaged in glaucoma patients; thus, they affect several functions of ipRGCs, including the PLR and circadian rhythms. Importantly, although a significant association was observed between daytime sleepiness and the PLR in this study, the correlation coefficient was not substantial $\left(R^{2}=0.080\right.$ for the correlation between the ESS score and peak PLR and $R^{2}=0.068$ for the correlation between the ESS score and the sustained PLR). Thus, important, unknown factors most likely contribute to this correlation. For example, the subjectivity of all self-report questionnaires might affect the final score. In addition, studies performed by Pack et al. and Gooneratne et al. showed that self-reported questionnaire of sleepiness can often be inaccurate when viewed alongside quantitative measures of alertness and sometimes is often viewed as a common and natural aspect of aging that can lead to a misinterpretation of the results $[38,39]$.

In addition, daytime sleepiness, in glaucoma group, was associated with a sustained PLR to the red flash. The peak and sustained PLRs to a red flash are well known to be correlated with the number and function of the rods and cones, not ipRGCs. One hypothesis explaining this finding is that rod and cones photoreceptors might also be affected in patients with chronic or advanced glaucoma, resulting in decreased signaling from these cells [40-42]. However, this topic remains controversial in the literature [40-43], and additional studies must be conducted to clarify the actual connection between the functions of ipRGCs and rod and cones photoreceptors.

Another interesting issue is the controversy concerning the relative preservation of ipRGCs among the diversity of RGCs [21, 44-47]. Li et al. showed that although the conventional RGC number was decreased in rats with increased IOP, the number of ipRGCs did not change. This result suggests that ipRGCs were resistant to the deleterious effects caused by increased IOP [45]. However, another study in mice 
demonstrated that ipRGCs appeared to be resistant to damage resulting from IOP elevation at an early age (approximately 5 months) but became vulnerable at a later age (approximately 11 months) [48]. The authors of that study suggested that the magnitude of IOP elevation required to damage ipRGCs must be greater than that required to induce RGC damage $[48,49]$. In addition, Drouyer et al. and de Zavalía et al. showed that glaucomatous rats and other rodents exhibited a delayed phase angle with respect to darkness $[46,47]$. These results are in agreement with ours, suggesting that glaucoma induces a circadian system dysfunction. One explanation for the differences between certain animal models and studies of patients with glaucoma is the endpoint evaluated, that is, morphological versusfunctional endpoints [49]. The primary focus of human studies has typically been ipRGC function, whereas the number and morphology of these cells have usually been considered in animal studies. The resistance mechanism that underlies the survival of ipRGCs is currently unknown. Although this question persists, our study analyzed two different functions involving ipRGCs in the same group of patients, and we found a strong association between them. Thus, our results strongly suggest that both non-imageforming functions of ipRGCs were affected.

Recently, Chen et al. demonstrated that there is not one specific type of ipRGC; rather, different subpopulations of ipRGCs exist with similar properties that innervate disparate brain regions to execute specific functions, such as the PLR and circadian photoentrainment [50]. In this study, pupil constriction, circadian oscillation (wheel running activity), the adjustment of the circadian clock to different light stimuli (i.e., circadian photoentrainment, "jet-lag" paradigms, phase shifting, and skeleton photoperiod), and the direct effect of constant and ultradian light on activity were measured [50]. The results showed that ipRGCs comprise functionally distinct subpopulations that differentially express a specific transcription factor [50]. ipRGCs that do not express this transcription factor innervate the suprachiasmatic nucleus of the hypothalamus, whereas ipRGCs that express this transcription factor innervate other known brain targets such as the olivary pretectal nucleus. Thus, the ipRGCs that express this transcription factor are associated with the PLR but not with circadian photoentrainment [50]. Because we did not evaluate this issue in the present study, future investigations should be conducted to elucidate the subpopulations of ipRGCs to determine how they are involved in non-imageforming functions.

The primary clinical finding of this study was that glaucoma patients had increased daytime sleepiness compared with healthy participants, and these results were associated with worse polysomnographic parameters and lower sustained PLRs to a blue flash with luminance of $250 \mathrm{~cd} / \mathrm{m}^{2}$. Therefore, circadian rhythm regulation, which is linked with ipRGCs' functions, should be evaluated in certain patients with glaucoma. Excessive daytime sleepiness is well known to affect quality of life, daytime function, and mortality; furthermore, previous studies of geriatric populations have revealed that excessive daytime sleepiness is associated with age, disability, dementia, and vision and hearing impairments [51].

This study has limitations. First, a relatively small number of patients were included in this study. Although future studies with greater numbers of patients should be undertaken to better understand the role of ipRGCs in sleep disorders, the present study did show strong agreement between the subjective and objective measures of sleep quality among patients with glaucoma. Second, sleep patterns are most likely affected by factors other than the hypothesized ipRGC loss associated with glaucoma. However, the subjective and objective measures of sleep quality (i.e., the ESS questionnaire and the polysomnographic parameters) strongly suggested that the damage to ipRGCs associated with glaucoma results in certain types of sleep disorders. Furthermore, regarding the primary endpoint of this study, we relied on self-reported histories of daytime sleepiness; it is possible that inaccuracies were present in patient recollection and reporting. However, we also used polysomnographic parameters (i.e., the gold standard test of sleep quality), and these parameters were in agreement with the ESS scores. In addition, because patient age is associated with a decrease in sleep quality and can affect PLR, this variable might have affected our final results $[16,51]$. Although the influence of age-related factors could not be completely excluded, our study did not reveal any significant differences in age between the two groups, and the multivariate analysis found that age was not a significant covariate. In addition, previous studies have shown that the postillumination response did not significantly decrease with age [52] and that only pupil diameter was reduced among the elderly population [52].

In conclusion, the results of this study demonstrated that patients with glaucoma had increased daytime sleepiness relative to healthy controls as measured by a selfreport questionnaire. In addition, daytime sleepiness was associated with a reduced sustained pupillary response and polysomnographic parameters. These ipRGC functions might be impaired in patients with glaucoma, thereby affecting their quality of life.

\section{Conflict of Interests}

The authors declare no conflict of interests regarding the present study.

\section{Acknowledgments}

This study was funded by FAPESP Thematic Project 2008/ 58731-2 (Dora Fix Ventura), a fellowship from the Brazilian National Research Council-CAPES 12309-13-3 (Carolina P. B. Gracitelli), FAPESP doctoral fellowship 2013/03553-0 (Gloria Liliana Duque-Chica), CAPES/PEC-PG 6160107 (Gloria Liliana Duque-Chica), and FAPESP (2009/54292-7) and CNPq (162576/2013-7) postdoctoral fellowships (Balazs V. Nagy). Dora Fix Ventura is the recipient of a $1 \mathrm{~A} \mathrm{CNPq}$ Productivity Grant. 


\section{References}

[1] J. F. Duffy and C. A. Czeisler, "Effect of light on human circadian physiology," Sleep Medicine Clinics, vol. 4, no. 2, pp. 165-177, 2009.

[2] R. J. Lucas, S. N. Peirson, D. M. Berson et al., "Measuring and using light in the melanopsin age," Trends in Neurosciences, vol. 37, no. 1, pp. 1-9, 2014.

[3] I. Provencio, I. R. Rodriguez, G. Jiang, W. P. Hayes, E. F. Moreira, and M. D. Rollag, "A novel human opsin in the inner retina," The Journal of Neuroscience, vol. 20, no. 2, pp. 600-605, 2000.

[4] D. M. Berson, F. A. Dunn, and M. Takao, "Phototransduction by retinal ganglion cells that set the circadian clock," Science, vol. 295, no. 5557, pp. 1070-1073, 2002.

[5] I. Provencio, H. M. Cooper, and R. G. Foster, "Retinal projections in mice with inherited retinal degeneration: implications for circadian photoentrainment," The Journal of Comparative Neurology, vol. 395, no. 4, pp. 417-439, 1998.

[6] Y. Fu, H. Zhong, M.-H. H. Wang et al., "Intrinsically photosensitive retinal ganglion cells detect light with a vitamin Abased photopigment, melanopsin," Proceedings of the National Academy of Sciences of the United States of America, vol. 102, no. 29, pp. 10339-10344, 2005.

[7] P. D. R. Gamlin, D. H. McDougal, J. Pokorny, V. C. Smith, K.-W. Yau, and D. M. Dacey, "Human and macaque pupil responses driven by melanopsin-containing retinal ganglion cells," Vision Research, vol. 47, no. 7, pp. 946-954, 2007.

[8] R. J. Lucas, S. Hattar, M. Takao, D. M. Berson, R. G. Foster, and K.-W. Yau, "Diminished pupillary light reflex at high irradiances in melanopsin-knockout mice," Science, vol. 299, no. 5604, pp. 245-247, 2003.

[9] B. Feigl and A. J. Zele, "Melanopsin-expressing intrinsically photosensitive retinal ganglion cells in retinal disease," Optometry and Vision Science, vol. 91, no. 8, pp. 894-903, 2014.

[10] R. N. Weinreb, T. Aung, and F. A. Medeiros, "The pathophysiology and treatment of glaucoma: a review," The Journal of the American Medical Association, vol. 311, no. 18, pp. 1901-1911, 2014.

[11] B. Feigl, D. Mattes, R. Thomas, and A. J. Zele, "Intrinsically photosensitive (melanopsin) retinal ganglion cell function in glaucoma," Investigative Ophthalmology and Visual Science, vol. 52, no. 7, pp. 4362-4367, 2011.

[12] C. Pérez-Rico, P. de la Villa, I. Arribas-Gómez, and R. Blanco, "Evaluation of functional integrity of the retinohypothalamic tract in advanced glaucoma using multifocal electroretinography and light-induced melatonin suppression," Experimental Eye Research, vol. 91, no. 5, pp. 578-583, 2010.

[13] L. Kankipati, C. A. Girkin, and P. D. Gamlin, "The postillumination pupil response is reduced in glaucoma patients," Investigative Ophthalmology \& Visual Science, vol. 52, no. 5, pp. 2287-2292, 2011.

[14] C. Nissen, B. Sander, D. Milea et al., "Monochromatic pupillometry in unilateral glaucoma discloses no adaptive changes subserved by the ipRGCs," Frontiers in Neurology, vol. 5, article $15,2014$.

[15] C. P. B. Gracitelli, G. L. Duque-Chica, A. L. Moura et al., "A positive association between intrinsically photosensitive retinal ganglion cells and retinal nerve fiber layer thinning in glaucoma," Investigative Ophthalmology \& Visual Science, vol. 55, no. 12, pp. 7997-8005, 2014.
[16] H. Wang, Y. Zhang, J. Ding, and N. Wang, "Changes in the circadian rhythm in patients with primary glaucoma," PLoS ONE, vol. 8, no. 4, Article ID e62841, 2013.

[17] C. P. B. Gracitelli, G. L. Duque-Chica, M. Roizenblatt et al., "Intrinsically photosensitive retinal ganglion cell activity is associated with decreased sleep quality in patients with glaucoma," Ophthalmology, vol. 122, no. 6, pp. 1139-1148, 2015.

[18] M. W. Johns, "A new method for measuring daytime sleepiness: the Epworth sleepiness scale," Sleep, vol. 14, no. 6, pp. 540-545, 1991.

[19] L. T. Chylack Jr., J. K. Wolfe, D. M. Singer et al., "The lens opacities classification system III," Archives of Ophthalmology, vol. 111, no. 6, pp. 831-836, 1993.

[20] J. C. Park, A. L. Moura, A. S. Raza, D. W. Rhee, R. H. Kardon, and D. C. Hood, "Toward a clinical protocol for assessing rod, cone, and melanopsin contributions to the human pupil response," Investigative Ophthalmology and Visual Science, vol. 52, no. 9, pp. 6624-6635, 2011.

[21] A. L. A. Moura, B. V. Nagy, C. La Morgia et al., "The pupil light reflex in leber's hereditary optic neuropathy: evidence for preservation of melanopsin-expressing retinal ganglion cells," Investigative Ophthalmology \& Visual Science, vol. 54, no. 7, pp. 4471-4477, 2013.

[22] R. Kardon, S. C. Anderson, T. G. Damarjian, E. M. Grace, E. Stone, and A. Kawasaki, "Chromatic pupil responses: preferential activation of the melanopsin-mediated versus outer photoreceptor-mediated pupil light reflex," Ophthalmology, vol. 116, no. 8, pp. 1564-1573, 2009.

[23] R. Kardon, S. C. Anderson, T. G. Damarjian, E. M. Grace, E. Stone, and A. Kawasaki, "Chromatic pupillometry in patients with retinitis pigmentosa," Ophthalmology, vol. 118, no. 2, pp. 376-381, 2011.

[24] A. Rechtschaffen and A. Kales, A Manual of Standardized Terminology, Techniques and Scoring System for Sleep Stages of Human Subjects, Brain Information Service, Brain Research Institute, University of California, Los Angeles, Los Angeles, Calif, USA, 1968.

[25] C. Iber, S. Ancoli-Israel, and A. L. Chesson Jr., The AASM Manual for the Scoring of Sleep and Associated Events: Rules, Terminology and Technical Specifications, American Academy of Sleep Medicine, Westchester, Ill, USA, 2007.

[26] C. A. Kushida, M. R. Littner, T. Morgenthaler et al., "Practice parameters for the indications for polysomnography and related procedures: an update for 2005," Sleep, vol. 28, no. 4, pp. 499521, 2005.

[27] I. E. Murdoch, S. S. Morris, and S. N. Cousens, "People and eyes: statistical approaches in ophthalmology," The British Journal of Ophthalmology, vol. 82, no. 8, pp. 971-973, 1998.

[28] l'Eclairage CId, Technical Note, Report on the First International Workshop on Circadian and Neurophysiological Photometry, Commission Internationale de l'Eclairage, Vienna, Austria, 2015.

[29] J. L. Ecker, O. N. Dumitrescu, K. Y. Wong et al., "Melanopsinexpressing retinal ganglion-cell photoreceptors: cellular diversity and role in pattern vision," Neuron, vol. 67, pp. 49-60, 2010.

[30] D. S. Mojon, C. W. Hess, D. Goldblum et al., "Normaltension glaucoma is associated with sleep apnea syndrome," Ophthalmologica, vol. 216, no. 3, pp. 180-184, 2002.

[31] G. Jean-Louis, F. Zizi, D. R. Lazzaro, and A. H. Wolintz, "Circadian rhythm dysfunction in glaucoma: a hypothesis," Journal of Circadian Rhythms, vol. 6, article 1, 2008. 
[32] O. Bergamin and R. H. Kardon, "Greater pupillary escape differentiates central from peripheral visual field loss," Ophthalmology, vol. 109, no. 4, pp. 771-780, 2002.

[33] R. H. Kardon, C. L. Haupert, and H. S. Thompson, "The relationship between static perimetry and the relative afferent pupillary defect," American Journal of Ophthalmology, vol. 115, no. 3, pp. 351-356, 1993.

[34] J. B. Kerrison, K. Buchanan, M. L. Rosenberg et al., "Quantification of optic nerve axon loss associated with a relative afferent pupillary defect in the monkey," Archives of Ophthalmology, vol. 119, no. 9, pp. 1333-1341, 2001.

[35] W.-D. A. Lagrèze and R. H. Kardon, "Correlation of relative afferent pupillary defect and estimated retinal ganglion cell loss," Graefe's Archive for Clinical and Experimental Ophthalmology, vol. 236, no. 6, pp. 401-404, 1998.

[36] H. Stanley Thompson, P. Montague, T. A. Cox, and J. J. Corbett, "The relationship between visual acuity, pupillary defect, and visual field loss," American Journal of Ophthalmology, vol. 93, no. 6, pp. 681-688, 1982.

[37] A. Kawasaki and R. H. Kardon, "Intrinsically photosensitive retinal ganglion cells," Journal of Neuro-Ophthalmology, vol. 27, no. 3, pp. 195-204, 2007.

[38] A. I. Pack, D. F. Dinges, P. R. Gehrman, B. Staley, F. M. Pack, and G. Maislin, "Risk factors for excessive sleepiness in older adults," Annals of Neurology, vol. 59, no. 6, pp. 893-904, 2006.

[39] N. S. Gooneratne, K. C. Richards, M. Joffe et al., "Sleep disordered breathing with excessive daytime sleepiness is a risk factor for mortality in older adults," Sleep, vol. 34, no. 4, pp. 435442, 2011.

[40] K. R. Kendell, H. A. Quigley, L. A. Kerrigan, M. E. Pease, and E. N. Quigley, "Primary open-angle glaucoma is not associated with photoreceptor loss," Investigative Ophthalmology \& Visual Science, vol. 36, no. 1, pp. 200-205, 1995.

[41] T. M. Nork, J. N. Ver Hoeve, G. L. Poulsen et al., "Swelling and loss of photoreceptors in chronic human and experimental glaucomas," Archives of Ophthalmology, vol. 118, no. 2, pp. 235245, 2000.

[42] S. Panda and J. B. Jonas, "Decreased photoreceptor count in human eyes with secondary angle-closure glaucoma," Investigative Ophthalmology and Visual Science, vol. 33, no. 8, pp. 25322536, 1992.

[43] Vaegan, S. L. Graham, I. Goldberg, L. Buckland, and F. C. Hollows, "Flash and pattern electroretinogram changes with optic atrophy and glaucoma," Experimental Eye Research, vol. 60, no. 6, pp. 697-706, 1995.

[44] C. La Morgia, F. N. Ross-Cisneros, A. A. Sadun et al., "Melanopsin retinal ganglion cells are resistant to neurodegeneration in mitochondrial optic neuropathies," Brain, vol. 133, no. 8, pp. 2426-2438, 2010.

[45] R. S. Li, B.-Y. Chen, D. K. Tay, H. H. L. Chan, M.-L. Pu, and K.-F. So, "Melanopsin-expressing retinal ganglion cells are more injury-resistant in a chronic ocular hypertension model," Investigative Ophthalmology and Visual Science, vol. 47, no. 7, pp. 2951-2958, 2006.

[46] N. de Zavalía, S. A. Plano, D. C. Fernandez et al., "Effect of experimental glaucoma on the non-image forming visual system," Journal of Neurochemistry, vol. 117, no. 5, pp. 904-914, 2011.

[47] E. Drouyer, O. Dkhissi-Benyahya, C. Chiquet et al., "Glaucoma alters the circadian timing system," PLoS ONE, vol. 3, no. 12, Article ID e3931, 2008.
[48] Q. Zhang, H. Vuong, X. Huang et al., "Melanopsin-expressing retinal ganglion cell loss and behavioral analysis in the ThylCFP-DBA/2J mouse model of glaucoma," Science China Life Sciences, vol. 56, no. 8, pp. 720-730, 2013.

[49] Q. Cui, C. Ren, P. J. Sollars, G. E. Pickard, and K. F. So, “The injury resistant ability of melanopsin-expressing intrinsically photosensitive retinal ganglion cells," Neuroscience, vol. 284, pp. 845-853, 2015.

[50] S.-K. Chen, T. C. Badea, and S. Hattar, "Photoentrainment and pupillary light reflex are mediated by distinct populations of ipRGCs," Nature, vol. 476, no. 7358, pp. 92-95, 2011.

[51] H. Frohnhofen, R. Popp, V. Willmann, H. C. Heuer, and A. Firat, "Feasibility of the Epworth Sleepiness Scale in a sample of geriatric in-hospital patients," Journal of Physiology and Pharmacology, vol. 60, supplement 5, pp. 45-49, 2009.

[52] L. Kankipati, C. A. Girkin, and P. D. Gamlin, "Post-illumination pupil response in subjects without ocular disease," Investigative Ophthalmology \& Visual Science, vol. 51, no. 5, pp. 2764-2769, 2010. 


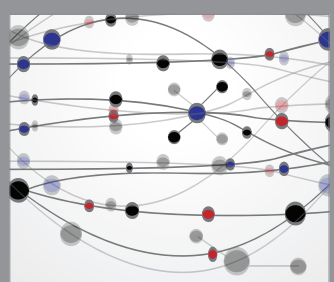

The Scientific World Journal
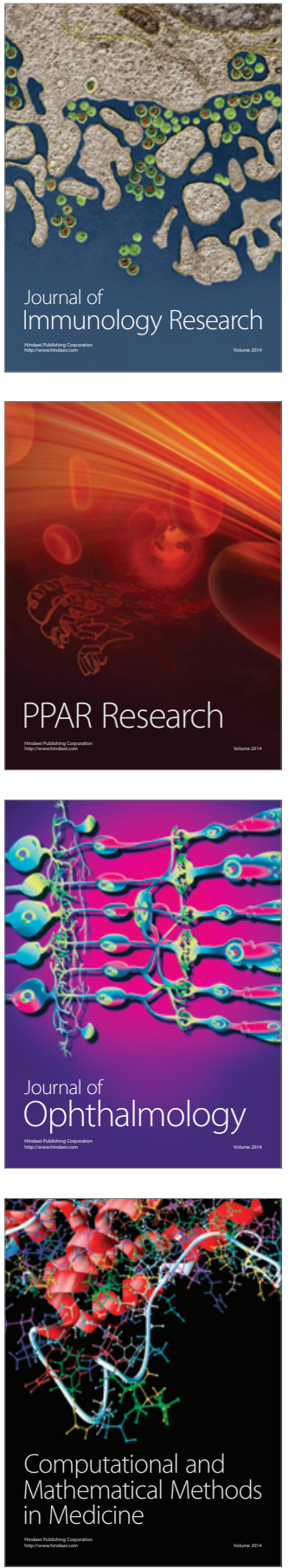

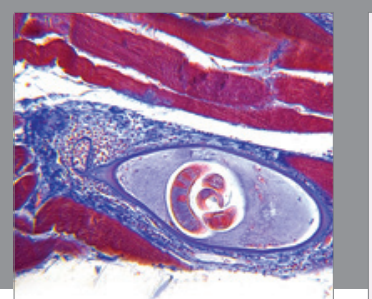

Gastroenterology Research and Practice

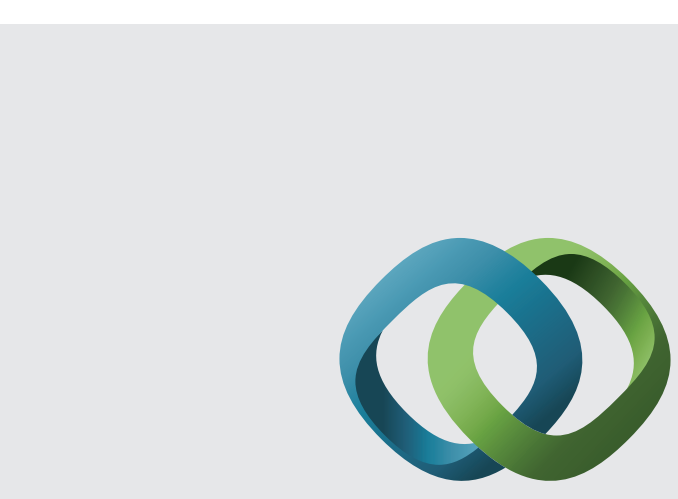

\section{Hindawi}

Submit your manuscripts at

http://www.hindawi.com
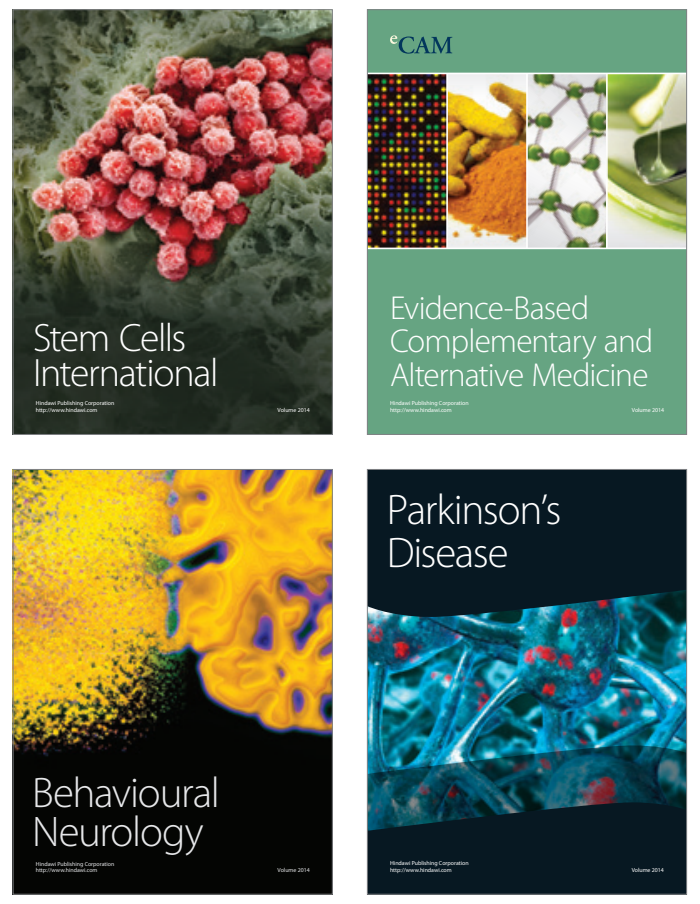
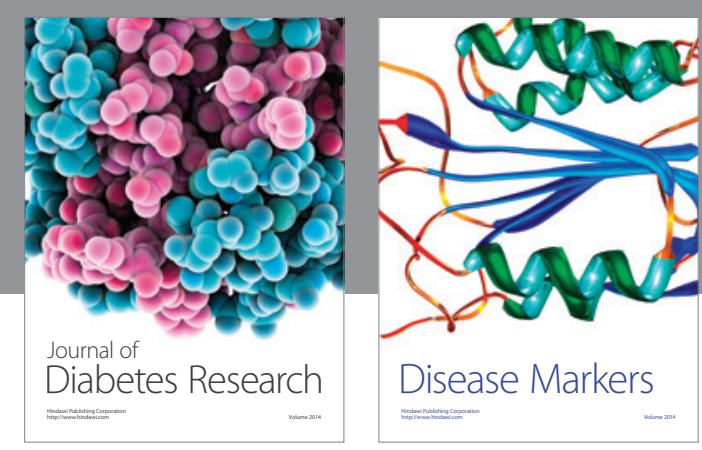

Disease Markers
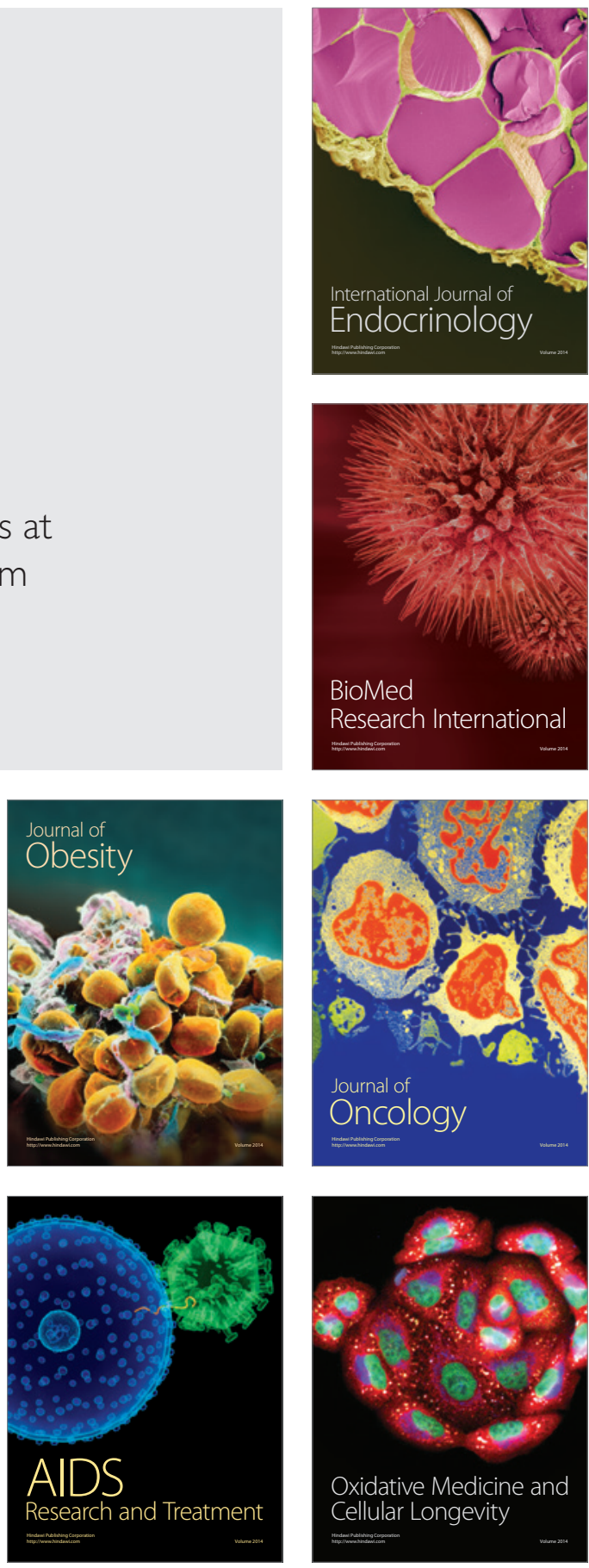